\title{
Salivary markers of oxidative stress and antioxidant status: Influence of external factors
}

\author{
Natália Kamodyováa ${ }^{\mathrm{a}}$ L’ubomíra Tóthováa ${ }^{\text {and Peter Celec }}$,a,c,* \\ ${ }^{a}$ Institute of Molecular Biomedicine, Comenius University, Bratislava, Slovakia \\ ${ }^{\mathrm{b}}$ Department of Molecular Biology, Comenius University, Bratislava, Slovakia \\ ${ }^{\mathrm{c}}$ Institute of Pathological Physiology, Comenius University, Bratislava, Slovakia
}

\begin{abstract}
.
BACKGROUND: Salivary markers of oxidative stress and antioxidant status represent promising tool for the research of oral diseases. One of the criteria is the validation of these biomarkers from the perspective of the confounding and modifying factors. AIM: To examine the effect of circadian rhythm, tooth-brushing and ascorbic acid treatment on selected salivary markers of oxidative and carbonyl stress, and antioxidant status.

SUBJECTS AND METHODS: Whole unstimulated saliva samples were collected from 19 healthy participants three times during a day, before and after tooth-brushing, and before and after the administration of vitamin C ( $250 \mathrm{mg}$ ). Advanced oxidation protein products (AOPP), thiobarbituric acid reactive substances (TBARS), advanced glycation end products (AGEs), ferric reducing antioxidant power (FRAP) and total antioxidant capacity (TAC) were measured.

RESULTS: Salivary AGEs levels varied significantly during the day $(p<0.05)$ with the highest concentrations in the morning. FRAP levels varied during the day $(p<0.01)$ with the highest concentrations in the afternoon. Tooth-brushing decreased AGEs $(p<0.05)$ and TBARS levels $(p<0.01)$ and increased FRAP levels $(p<0.05)$. Single intake of vitamin C significantly decreased AGEs $(p<0.001)$ and increased both FRAP $(p<0.01)$ and TAC $(p<0.01)$ concentrations.

CONCLUSION: Significant daily variations were observed in salivary AGEs and FRAP levels. Tooth-brushing and treatment with vitamin $\mathrm{C}$ decreased carbonyl stress and increased the antioxidant status. These results are important from the perspective of using saliva for the research of oral diseases.
\end{abstract}

Keywords: Oxidative stress, saliva, antioxidant status, circadian rhythm, tooth-brushing, ascorbic acid

\section{Introduction}

Reactive oxygen species (ROS) and reactive nitrogen species (RNS) play an important role in physiological conditions in cell signaling and metabolic pathways. On the other hand, decreased antioxidant levels and/or increased production of reactive metabolites can lead to the destruction of homeostasis and drive the ox-

*Corresponding author: Peter Celec, Institute of Molecular Biomedicine, Comenius University, Sasinkova 4, 81108 Bratislava, Slovakia. Tel.: +421 2 59357371; Fax: +4212 59357631; E-mail: petercelec@gmail.com. idative damage $[3,16]$. Oxidative/nitrosative stress can seriously influence the cell viability and induce cellular responses leading to cell death [7]. Many studies have shown connection between oxidative damage of molecules and patophysiological mechanisms of severe diseases like atherosclerosis [14], neurodegenerative diseases [15] and diabetes [41]. There is also a relationship between oxidative stress and aging processes [49].

In association with oral and dental diseases (especially periodontitis), changed oxidative stress markers in saliva were reported [2,46,51]. Being a noninvasive biofluid, saliva allows easy, noninvasive and safe sam- 
ple collection. It is therefore suitable for monitoring of general health and disease in children, elderly people, and non-collaborative subjects. Salivary analysis is very attractive due to the possibility of repeated sampling and longitudinal monitoring $[22,25]$. To use the potential of salivary oxidative stress markers for diagnostics of oral diseases, various criteria must be optimized.

Three main groups of endproducts are used as biomarkers of oxidative damage: - lipid peroxidation products, oxidized proteins and products of DNA oxidation and fragmentation [40]. One of the main validation criteria of a biomarker is the influence of confounding and modifying factors [7]. As reported previously, the limitations for assessing salivary thiobarbituric acid reacting substances (TBARS) - markers of lipoperoxidation- are the external influences and timedependent variations [18]. To our knowledge, the influence of external factors on salivary biomarkers of oxidative stress has not been examined besides this study. Thus, the aim of our study was to question the effect of circadian rhythm, tooth-brushing, and treatment with vitamin $\mathrm{C}$ on selected salivary biomarkers of oxidative stress and antioxidant status. In the presented work, advanced oxidation protein products (AOPP) - markers of oxidative damage to proteins, thiobarbituric acid reactive substances (TBARS) markers of lipoperoxidation, and advanced glycation end products (AGEs) markers of carbonyl stress were analyzed. The antioxidant status was assessed using two assays - ferric reducing antioxidant power (FRAP) and total antioxidant capacity (TAC).

\section{Subjects and methods}

\subsection{Subjects and sampling}

Whole unstimulated saliva samples were obtained from 19 young healthy probands ( 9 females and 10 males) aged 20-30 (25.9 \pm 2.5$)$ years during two consecutive days. On the first day, the sampling was performed at 08:00, 14:00 and 21:00 before toothbrushing. An additional sampling was carried in the morning 10 minutes after tooth-brushing. On the following day, the saliva samples were collected before and 30 minutes after an oral administration of $250 \mathrm{mg}$ of vitamin C. Specifically, powder vitamin $\mathrm{C}$ was dissolved in water to final concentration $125 \mathrm{~g} / \mathrm{L}$. The probands were asked to drink $2 \mathrm{~mL}$ of this vitamin $\mathrm{C}$ solution provided in microtube (Eppendorf, Ham- burg, Germany). One male proband was excluded from analyses before and after vitamin $\mathrm{C}$, because he did not provide saliva sample after vitamin $\mathrm{C}$ consumption. Collected saliva samples were stored at $-20^{\circ} \mathrm{C}$ until analyses were performed. All saliva samples were collected after rinsing the mouth with water and the volunteers were instructed not to eat or drink $30 \mathrm{~min}$ utes before any sampling. Smokers and people with systemic disorders were excluded from the study. The study was approved by the Ethics committee of the Institute of Molecular Biomedicine, Comenius University in Bratislava, Slovakia. The volunteers gave informed consent for participation in the study. All reagents or chemicals used in our experiments were purchased from Sigma-Aldrich, Steinheim, Germany.

\subsection{AOPP determination}

Salivary AOPP levels were determined using a spectrophotometric method by Witko-Sarsat et al. [18]. Briefly, $200 \mu \mathrm{L}$ of saliva was incubated with $20 \mu \mathrm{L}$ of glacial acetic acid. The absorbance was read immediately at $340 \mathrm{~nm}$. AOPP concentration was expressed in $\mu \mathrm{mol} / \mathrm{L}$ on the basis of the calibration curve of chloramin $\mathrm{T}$ with potassium iodide.

\subsection{AGEs determination}

Salivary AGEs were assessed using spectrofluorometric method $(\lambda$ ex. $=370 \mathrm{~nm}, \lambda$ em. $=440 \mathrm{~nm})$ according to Münch et al. [34]. Saliva samples were diluted 10-fold with PBS (phosphate buffered saline, $\mathrm{pH}$ 7.2) and the specific fluorescence of AGEs was expressed in arbitrary units.

\subsection{FRAP determination}

Salivary FRAP levels were measured according to Benzie and Strain [20]. Two hundred $\mu \mathrm{L}$ of prewarmed $37^{\circ} \mathrm{C}$ FRAP reagent (1 volume of $3 \mathrm{~mol} / \mathrm{L}$ acetate buffer, pH $3.6+1$ vol of $10 \mathrm{mmol} / \mathrm{L} \mathrm{2,4,6-}$ tripyridyl-S-triazine in $40 \mathrm{mmol} / \mathrm{L} \mathrm{HCl}+1$ vol of $20 \mathrm{mmol} / \mathrm{L} \mathrm{FeCl}_{3}$ ) was mixed with $20 \mu \mathrm{L}$ of saliva. Absorbance was read at $593 \mathrm{~nm}$. Ferrous sulphate was used as standard and the concentration of FRAP was expressed in $\mu \mathrm{mol} / \mathrm{L}$.

\subsection{TAC determination}

Salivary TAC was determined according to Erel [19]. Saliva was mixed with $0.4 \mathrm{~mol} / \mathrm{L}$ acetate buffer $(\mathrm{pH}$ 
5.8), incubated with ABTS (2,2'-azino-bis (3-ethylbenzthiazoline-6-sulphonic acid)) and oxidized with hydrogen peroxide in $0.03 \mathrm{~mol} / \mathrm{L}$ acetate buffer $(\mathrm{pH}$ 3.6). Absorbance was taken at $660 \mathrm{~nm}$. TAC was expressed in $\mu \mathrm{mol} / \mathrm{L}$ on the basis of the calibration curve of trolox.

\subsection{TBARS determination}

Salivary TBARS levels were determined using the spectrofluorometric method according to Behuliak et al. [4]. Twenty $\mu \mathrm{L}$ of saliva was derivatized with $0.67 \%$ thiobartituric acid in acidic medium of acetic acid $\left(95^{\circ} \mathrm{C}, 45 \mathrm{~min}\right)$, extracted with n-butanol, and measured at $\lambda$ ex. $=515 \mathrm{~nm}, \lambda$ em. $=535 \mathrm{~nm}$. TBARS concentration was expressed in $\mu \mathrm{mol} / \mathrm{L}$ on the basis of 1,1,3,3-tetraethoxypropane standard.

\subsection{Total proteins determination}

Proteins were quantified using BCA protein assay kit (Sigma Aldrich, Steinheim, Germany). Briefly 10 $\mu \mathrm{L}$ of saliva was mixed with $200 \mu \mathrm{L}$ of BCA working reagent, incubated $30 \mathrm{~min}$ at $37^{\circ} \mathrm{C}$ and measured at 562 $\mathrm{nm}$. Levels of salivary oxidative stress markers were normalized for total proteins.

\subsection{Statistical analysis}

Repeated measures ANOVA, Tukey's multiple comparison test and paired t-test were used for statistical analysis of obtained data. Analysis was performed with XLStatistics 10.05.30 and GraphPad Prism 5.03. Data were presented as mean $\pm \mathrm{SD}$.

\section{Results and discussion}

No significant differences in salivary levels of oxidative stress markers were observed between men and women. Based on these results, data from both genders were combined for further analyses.

\subsection{Effect of circadian rhythm}

The results showed significantly higher levels of salivary AGEs in the morning (by 76\%, $p<0.05$, Tukey's test, Fig. 1A). The levels of salivary AOPP did not differ significantly during the day (Fig. 1B).
TBARS concentrations were highest in the morning hours, although the differences between morning and afternoon concentrations were not statistically significant (Fig. 1C). Previously, significantly highest TBARS levels were observed in the morning hours [18]. On the other hand $\mathrm{Su}$ et al. observed diurnal variations in salivary protein carbonyl concentrations with highest concentrations at 2:00 p.m. [50]. Significant daily variations were observed in salivary FRAP levels ( $F=3.019, p<0.01$, ANOVA) representing antioxidant status. FRAP concentrations were lowest in the morning and were significantly higher in the afternoon (by 53\%, $p<0.01$, Tukey's test, Fig. 1D). It is interesting that the antioxidant profile expressed using TAC assay did not differ significantly during the day (Fig. 1E). The difference between the FRAP and TAC assays might have occurred due to the fact that the FRAP assay measures primarily the nonprotein total antioxidant capacity while TAC assay developed by Erel [11] measures the antioxidative effect of proteins as well, which has an important contribution to antioxidant status of saliva. The increase in FRAP levels could be the consequence of fluctuations in amount of low molecular antioxidants. In contrast to our results, Borisenkov et al. [6] reported circadian variations in salivary TAC concentrations with maximum at 6.00 a.m. and minimum at 3 p.m. and suggested that the influence of melatonin in the morning increases TAC. Melatonin, an important circadian marker which synchronizes the internal hormonal environment according to the light-dark cycle, has been proved to be an effective antioxidant $[31,44]$. Melatonin is measurable in saliva and significant correlation was reported between melatonin concentrations in blood and saliva [35]. The study of Benot et al. [5] reported the impact of melatonin on total antioxidant status (TAS) in human serum with maximal values of TAS at 1:00 a.m. which was similar to our study and contrasted the study of Borisenkov et al. [6]. The TAS levels were not increased at 7.00 a.m. [5]. The contribution of individual antioxidants to salivary antioxidant status should be assessed in detail in future experiments.

The nocturnal levels of markers were not analyzed in our study. Although the nocturnal levels would be interesting in terms of monitoring circadian rhythm, the perspective of diagnostics are important mainly the diurnal levels. The circadian variations in salivary concentrations of other individual antioxidants were not examined in previous studies. The limitation of our study is that salivary flow rates were not assessed dur- 

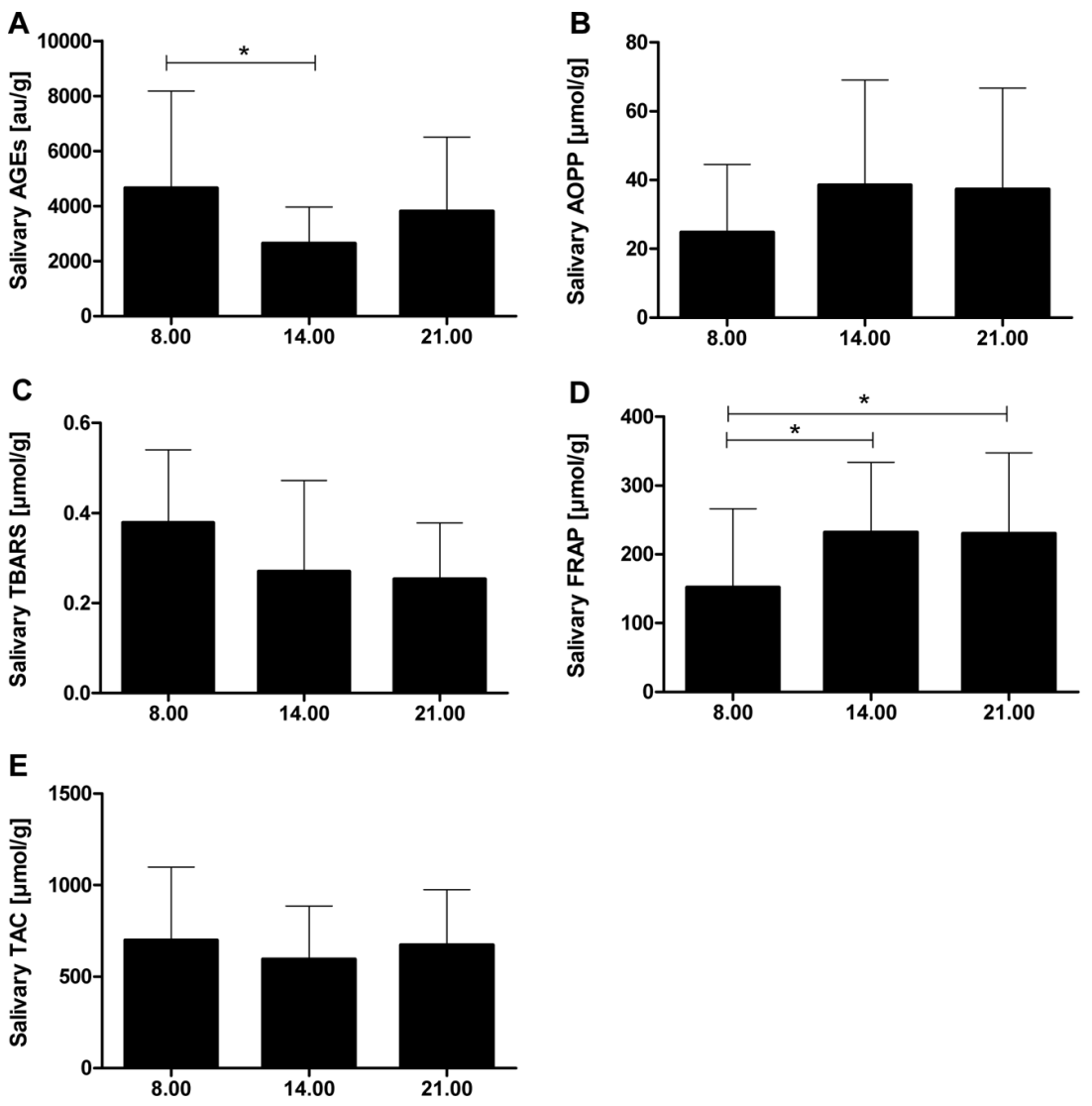

Fig. 1. Effect of daily variations on salivary markers of oxidative stress and antioxidant status. (A) Salivary AGEs levels, biomarker of carbonyl stress. (B) Salivary AOPP levels, biomarker of oxidative damage to proteins. (C) Salivary TBARS levels, biomarker of lipid peroxidation. (D) Salivary FRAP levels, biomarker of antioxidant status. (E) Salivary TAC levels, biomarker of antioxidant status. Data are presented as mean + $\mathrm{SD},{ }^{*}$ denotes $p<0.05$

ing saliva collection. Salivary flow rates could vary in relation to circadian cycle, individual hydration, food stimulation, oral hygiene, etc. [8]. Normalization to total proteins is used as alternative to salivary flow to look for differences in the ratio of biochemical analytes present in saliva $[12,26]$. As in previous studies on salivary oxidative stress markers [9,37], we have decided to normalize levels of salivary oxidative stress markers to total proteins.

The knowledge of circadian variations in salivary biomarkers of oxidative stress is important for designing the collection of samples, especially in studies oriented on oral and other systemic diseases. Assessment of circadian rhythm could be also valuable for optimization of therapeutic interventions according to expected redox homeostasis [50].

\subsection{Effect of tooth-brushing}

Cleaning the teeth decreased the AGEs levels in 12 out of 19 probands on average by $46 \%(p<0.05$, paired t-test, Fig. 2A). Salivary AOPP concentrations were higher in 16 out of 19 probands after toothbrushing on average by $56 \%(p<0.05$, paired $\mathrm{t}-$ test, Fig. 2B). The effect of cleaning the teeth on salivary AOPP levels is rather surprising. On the other hand these data look to be in agreement with recently published review in which AOPP concentrations are suggested as a measure of non-enzymatic antioxidant proteins such as oxidized fibrinogen, at least in plasma [48]. Significant decrease in salivary TBARS after tooth-brushing was reported previously by our group and the local origin of salivary TBARS and oxidative stress were hypothesized [18]. Presented results are in accordance to our previous study [18]. Salivary TBARS concentrations were significantly lower after tooth-brushing in 17 out of 19 probands (by $42 \%$, $p<0.01$, paired t-test, Fig. 2C). The similar trend in reduction of salivary TBARS and AGEs levels is probably caused by close link between oxidative and carbonyl stress. Reactive carbonyl compounds formed 

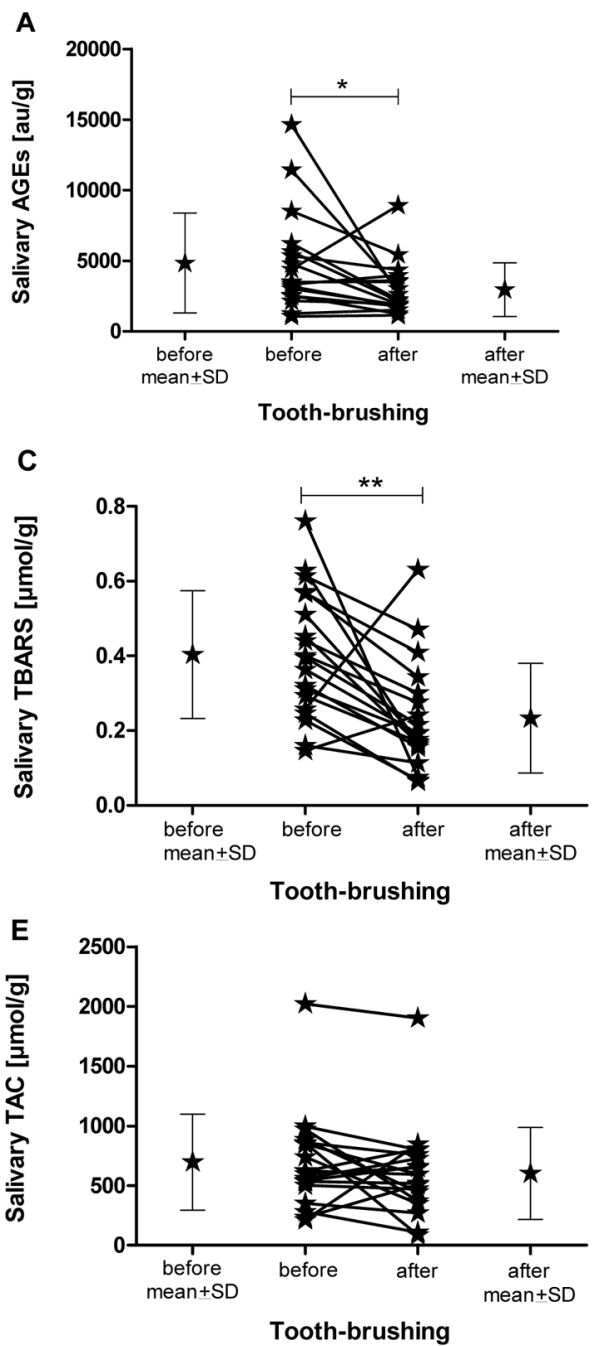

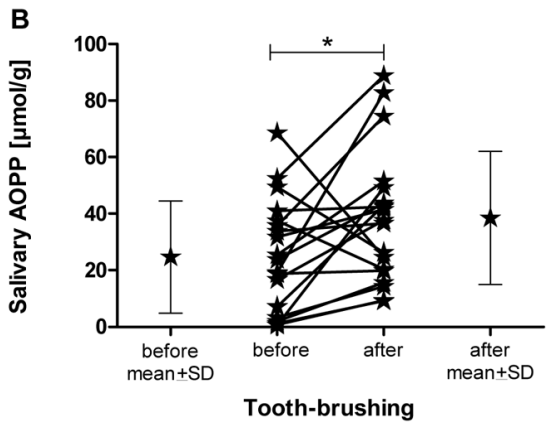

D

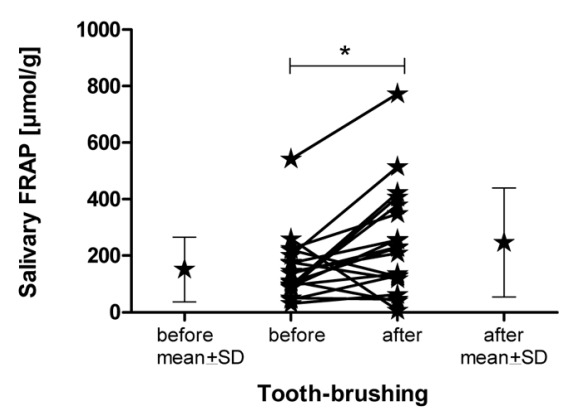

Fig. 2. Effect of tooth-brushing on salivary markers of oxidative stress and antioxidant status. (A) Salivary AGEs levels, biomarker of carbonyl stress. (B) Salivary AOPP levels, biomarker of oxidative damage to proteins. (C) Salivary TBARS levels, biomarker of lipid peroxidation. (D) Salivary FRAP levels, biomarker of antioxidant status. (E) Salivary TAC levels, biomarker of antioxidant status. Data are presented as mean \pm $\mathrm{SD},{ }^{* *}$ denotes $p<0.01$ and ${ }^{*} p<0.05$.

from carbohydrates react nonenzymaticaly with protein amino groups and yield AGEs during the Maillard reaction. Reactive carbonyl compounds, e.g. glyoxal, malodialdehyde, hydroxynonenal, reacting with amino groups in proteins are also produced during lipid peroxidation [32]. Significant increase was detected in salivary FRAP after tooth-brushing in 14 out of 19 probands on average by $89 \%$ ( $p<0.05$, paired t-test, Fig. 2D) but analogously to the influence of daily variations, the salivary TAC levels were not significantly influenced (Fig. 2E). Salivary TAC was decreased in 13 out of 19 probands after tooth-brushing.

Several studies have addressed the role of oxidative stress in the pathology of oral and dental dis- eases, especially periodontitis $[1,2,13,54]$ and the hypothesis that good oral hygiene may decrease oxidative stress in saliva was proposed [53]. In our study, saliva samples collected before and 10 minutes after tooth-brushing were monitored. Traditionally, toothbrushing is viewed as procedure for mechanical removing of dental plaque [23]. The removal of bacteria potentially producing free radicals or reduced response of host inflammatory system could lead to decreased oxidative stress in saliva. On the other hand, more recent studies indicate that mechanical stimulation promotes cell proliferation of basal cells and fibroblasts and reduces inflammatory processes, rather than removal of periodontal pathogens $[10,20]$. The effect 

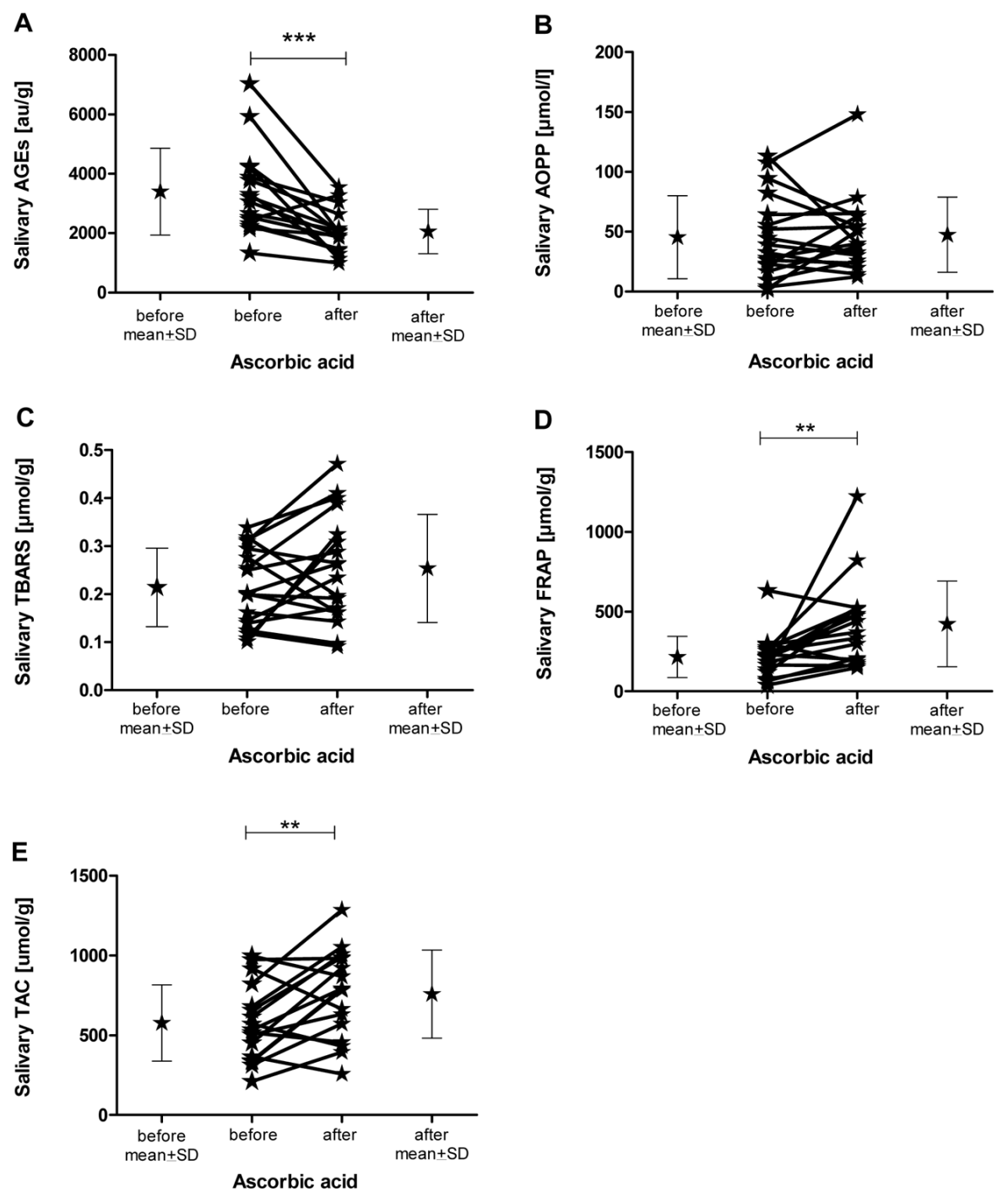

Fig. 3. Effect of ascorbic acid treatment on salivary markers of oxidative stress and antioxidant status. (A) Salivary AGEs levels, biomarker of carbonyl stress. (B) Salivary AOPP levels, biomarker of oxidative damage to proteins. (C) Salivary TBARS levels, biomarker of lipid peroxidation. (D) Salivary FRAP levels, biomarker of antioxidant status. (E) Salivary TAC levels, biomarker of antioxidant status. Data are presented as mean $\pm \mathrm{SD},{ }^{* * *}$ denotes $p<0.001$ and ${ }^{* *} p<0.01$.

of bacterial flora on salivary oxidative stress should be examined in further studies. Mechanical toothbrushing led to decreased 8-hydroxydeoxyguanosine levels (marker of oxidative DNA damage) in periodontitis rat model. The study demonstrated decreased oxidative damage in gingiva and potential contribution to reduced circulating oxidative stress [10]. Higher antioxidant values may also be caused by expulsion of gingival crevicular fluid following tooth-brushing and artificially increased antioxidant concentration in saliva [21]. Hoek et al. has reported increased albumin levels even more than 45 minutes after toothbrushing [19]. Albumin is an important salivary antiox- idant [33] and may therefore represent another factor contributing to increased total antioxidant capacity after tooth-brushing.

Based on our results it can be concluded that brushing of teeth can significantly influence oxidative stress markers and result in bias in diagnostic tests. To avoid this problem another study should explore the concentrations of salivary oxidative stress markers at different time points after tooth-brushing. Based on these results the optimal time after tooth-brushing should be set for collection of saliva. On the other hand, better parodontal status in periodontitis patients with better oral hygiene [53] could explain decreased oxidative stress as 
the result of better oral hygiene. One of the drawbacks of our study is that oral hygiene and health status of the subjects were not determined.

\subsection{Effect of vitamin $C$}

Single dose of vitamin C (250 mg) induced significant decrease in AGEs levels in 17 out of 18 probands on average by $64 \%$ ( $p<0.001$, paired t-test, Fig. 3A). No significant changes were detected in AOPP levels (Fig. 3B). Salivary TBARS levels were not significantly influenced after vitamin $C$ treatment. It is interesting that tendency to higher levels of TBARS concentrations after vitamin $\mathrm{C}$ was detected in 10 out of 18 probands. In our previous study, significantly decreased TBARS concentrations were observed after ascorbic acid treatment [18]. An increase in salivary FRAP was observed in 14 out of 18 probands on average by $107 \%$ ( $p<0.01$, paired t-test, Fig. 3D) and TAC in 12 out of 18 probands on average by $1 \%(p<0.01$, paired t-test, Fig. 3E). Vitamin $\mathrm{C}$ is free radical scavenging nonenzymatic antioxidant. Vitamin $\mathrm{C}$ also acts as cofactor for enzymes involved in collagen hydroxylation, biosynthesis of carnitine and norepinephrine, tyrosine metabolism, and amidation of peptide hormones [28]. Vitamin C works directly as free radical scavenger in watery environment of the cells and by interacting with vitamin $\mathrm{E}$ in the lipid rich areas of the cells [45]. It was shown that plasma vitamin C is lowered in chronic or acute oxidant states $[24,30,36]$. Only few studies have monitored concentrations and vitamin C supplementation in saliva [17,39,42,43,45,47]. Requirement for vitamin $\mathrm{C}$ is in humans satisfied by the ordinary diet [29]. Our results have shown that vitamin C supplementation led to significantly decreased carbonyl stress and increased antioxidant status. The conditions of saliva samples collection for oxidative stress monitoring should be standardized in terms of diet and vitamin supplementation. On the other hand, vitamin C supplementation could be potentially used for antioxidative therapy of oral diseases in which increased oxidative stress was reported. Orally administered vitamin C led to decreased 8-hydroxydeoxyguanosine, improved glutathione ratio, and decreased expression of $\operatorname{IL} 1 \alpha$ and $\operatorname{IL} 1 \beta$ in experimental rat periodontitis model [52]. In depletion-supplementation study in healthy men significantly improved gingival index after supplementation with $600 \mathrm{mg} /$ day of vitamin $\mathrm{C}$ and this effect was reversed during the following period of depletion to $5 \mathrm{mg} /$ day. In addition, bleeding scores decreased significantly after the supplementation period and increased during the period of depletion [27].
One of the limitations of our study is that the concentrations of vitamin $\mathrm{C}$ before and after vitamin $\mathrm{C}$ supplementation were not monitored in saliva. Plasma concentration of vitamin $\mathrm{C}$ are highly controlled when the vitamin is taken orally [38]. But no pharmacokinetic studies on vitamin $C$ were conducted in saliva. In the future it would be beneficial to perform dose concentration, dynamics and long-term studies of salivary vitamin $\mathrm{C}$ in relation to oxidative stress biomarkers.

\subsection{Conclusion}

Salivary carbonyl stress and antioxidant status are influenced by daily variations. Tooth-brushing and treatment with vitamin $\mathrm{C}$ decrease carbonyl stress and increase antioxidant status. The results are important for further research on the role of oxidative and carbonyl stress in the pathogenesis of oral diseases and for the potential use of salivary markers of oxidative and carbonyl stress in the diagnostics of oral diseases, at least on a population level.

\section{Acknowledgements}

The study was supported by Slovak Research and Development Agency (grant VMSP-II-0027-09).

\section{References}

[1] F. Agha-Hosseini, I. Mirzaii-Dizgah, S. Mikaili, and M. Abdollahi, Increased salivary lipid peroxidation in human subjects with oral lichen planus, Int J Dent Hyg 7 (2009), 246250.

[2] F.A. Akalin, E. Baltacioglu, A. Alver, and E. Karabulut, Lipid peroxidation levels and total oxidant status in serum, saliva and gingival crevicular fluid in patients with chronic periodontitis, J Clin Periodontol 34 (2007), 558-565.

[3] C. Arana, A. Cutando, M.J. Ferrera, G. Gómez-Moreno, C.V. Worf, M.J. Bolaños, G. Escames, and D. Acuña-Castroviejo, Parameters of oxidative stress in saliva from diabetic and parenteral drug addict patients, J Oral Pathol Med 35 (2006), 554-559.

[4] M. Behuliak, R. Palffy, R. Gardlik, J. Hodosy, L. Halcak, and P. Celec, Variability of thiobarbituric acid reacting substances in saliva, Dis Markers 26 (2009), 49-53.

[5] S. Benot, R. Goberna, R.J. Reiter, S. Garcia-Maurino, C. Osuna, and J.M. Guerrero, Physiological levels of melatonin contribute to the antioxidant capacity of human serum, $J$ Pineal Res 27 (1999), 59-64.

[6] M. Borisenkov, L. Erunova, E. Lyuseva, and N. Pozdeeva, Diurnal changes in the total antioxidant activity of human saliva, Hum Physiol 33 (2007), 375-376.

[7] I. Dalle-Donne, R. Rossi, R. Colombo, D. Giustarini, and A. Milzani, Biomarkers of oxidative damage in human disease, Clin Chem 52 (2006), 601-623. 
[8] C. Dawes, Circadian rhythms in human salivary flow rate and composition, J Physiol 220 (1972), 529-545.

[9] R. Deminice, T. Sicchieri, P.O. Payao, and A.A. Jordao, Blood and salivary oxidative stress biomarkers following an acute session of resistance exercise in humans, Int J Sports Med 31 (2010), 599-603.

[10] D. Ekuni, T. Tomofuji, N. Tamaki, T. Sanbe, T. Azuma, R. Yamanaka, T. Yamamoto, and T. Watanabe, Mechanical stimulation of gingiva reduces plasma $8-\mathrm{OHdG}$ level in rat periodontitis, Arch Oral Biol 53 (2008), 324-329.

[11] O. Erel, A novel automated direct measurement method for total antioxidant capacity using a new generation, more stable ABTS radical cation, Clin Biochem 37 (2004), 277-285.

[12] J. Furuholm, T. Sorsa, M. Qvarnstrom, S.J. Janket, T. Tervahartiala, P. Nuutinen, and J.H. Meurman, Salivary matrix metalloproteinase- 8 in patients with and without coronary heart disease may indicate an increased susceptibility to periodontal disease, J Periodontal Res 41 (2006), 486-489.

[13] A. Guentsch, P. Preshaw, S. Bremer-Streck, G. Klinger, E. Glockmann, and B. Sigusch, Lipid peroxidation and antioxidant activity in saliva of periodontitis patients: effect of smoking and periodontal treatment, Clin Oral Investig 12 (2008), 345-352.

[14] M. Haidari, M. Ali, L. Gangehei, M. Chen, W. Zhang, and M.I. Cybulsky, Increased oxidative stress in atherosclerosispredisposed regions of the mouse aorta, Life Sci 87 (2010), 100-110.

[15] B. Halliwell, Role of free radicals in the neurodegenerative diseases - Therapeutic implications for antioxidant treatment, Drugs Aging 18 (2001), 685-716.

[16] B. Halliwell and M. Whiteman, Measuring reactive species and oxidative damage in vivo and in cell culture: how should you do it and what do the results mean? Br J Pharmacol 142 (2004), 231-255

[17] N. Hegde, S.N. Suchetha Kumari, M.N. Hegde, P.M. Chandra, and Nireeksha, Lipid peroxidation and Vitamin $\mathrm{C}$ levels in saliva of oral precancerous patients- an In-vitro study, Res $J$ Pharm Biol Chem Sci 2 (2011).

[18] J. Hodosy and P. Celec, Daytime of sampling, tooth-brushing and ascorbic acid influence salivary thiobarbituric acid reacting substances - A potential clinical marker of gingival status, Dis Markers 21 (2005), 203-207.

[19] G.H. Hoek, H.S. Brand, E.C. Veerman, and A.V. Amerongen, Toothbrushing affects the protein composition of whole saliva, Eur J Oral Sci 110 (2002), 480-481

[20] M. Horiuchi, T. Yamamoto, T. Tomofuji, A. Ishikawa, M. Morita, and T. Watanabe, Toothbrushing promotes gingival fibroblast proliferation more effectively than removal of dental plaque, J Clin Periodontol 29 (2002), 791-795.

[21] I.L.C. Chapple, G.I. Mason, I. Garner, J.B. Matthews, G.H. Thorpe, S.R.J. Maxwell, and T.P. Whitehead, Enhanced chemiluminescent assay for measuring the total antioxidant capacity of serum, saliva and crevicular fluid, Ann Clin Biochem 34 (1997), 412-421.

[22] S. Chiappin, G. Antonelli, R. Gatti, and E.F. De Palo, Saliva specimen: A new laboratory tool for diagnostic and basic investigation, Clin Chim Acta 383 (2007), 30-40.

[23] N.P. Lang, B.R. Cumming, and H. Loe, Toothbrushing frequency as it relates to plaque development and gingival health, J Periodontol 44 (1973), 396-405.

[24] M. Langlois, D. Duprez, J. Delanghe, M. De Buyzere, and D.L. Clement, Serum vitamin C concentration is low in peripheral arterial disease and is associated with inflammation and severity of atherosclerosis, Circulation 103 (2001), 18631868.

[25] J.M. Lee, E. Garon, and D.T. Wong, Salivary diagnostics, Orthod Craniofac Res 12 (2009), 206-211.

[26] J.Y. Lee, J.W. Chung, Y.K. Kim, S.C. Chung, and H.S. Kho, Comparison of the composition of oral mucosal residual saliva with whole saliva, Oral Dis 13 (2007), 550-554.

[27] P.J. Leggott, P.B. Robertson, D.L. Rothman, P.A. Murray, and R.A. Jacob, The effect of controlled ascorbic acid depletion and supplementation on periodontal health, J Periodontol 57 (1986), 480-485.

[28] M. Levine, S.C. Rumsey, R. Daruwala, J.B. Park, and Y. Wang, Criteria and recommendations for vitamin $\mathrm{C}$ intake, JAMA 281 (1999), 1415-1423.

[29] J. Mandl, A. Szarka, and G. Banhegyi, Vitamin C: Update on physiology and pharmacology, Br J Pharmacol 157 (2009), 1097-1110.

[30] C.R. Mayland, M.I. Bennett, and K. Allan, Vitamin C deficiency in cancer patients, Palliat Med 19 (2005), 17-20.

[31] D.K. Mirick and S. Davis, Melatonin as a biomarker of circadian dysregulation, Cancer Epidemiol Biomarkers Prev 17 (2008), 3306-3313

[32] T. Miyata, K. Kurokawa, and C. Van Ypersele De Strihou, Advanced glycation and lipoxidation end products: role of reactive carbonyl compounds generated during carbohydrate and lipid metabolism, J Am Soc Nephrol 11 (2000), 1744-1752.

[33] S. Moore, K.A. Calder, N.J. Miller, and C.A. Rice-Evans, Antioxidant activity of saliva and periodontal disease, Free Radic Res 21 (1994), 417-425.

[34] G. Munch, R. Keis, A. Wessels, P. Riederer, U. Bahner, A. Heidland, T. Niwa, H.D. Lemke, and R. Schinzel, Determination of advanced glycation end products in serum by fluorescence spectroscopy and competitive ELISA, Eur J Clin Chem Clin Biochem 35 (1997), 669-677.

[35] R. Nowak, I.C. McMillen, J. Redman, and R.V. Short, The correlation between serum and salivary melatonin concentrations and urinary 6-hydroxymelatonin sulphate excretion rates: Two non-invasive techniques for monitoring human circadian rhythmicity, Clin Endocrinol (Oxf) 27 (1987), 445452 .

[36] K. Nyyssonen, M.T. Parviainen, R. Salonen, J. Tuomilehto, and J.T. Salonen, Vitamin C deficiency and risk of myocardial infarction: Prospective population study of men from eastern Finland, BMJ 314 (1997), 634-638.

[37] L.K. Ozturk, H. Furuncuoglu, M.H. Atala, O. Ulukoylu, S. Akyuz, and A. Yarat, Association between dental-oral health in young adults and salivary glutathione, lipid peroxidation and sialic acid levels and carbonic anhydrase activity, Braz $J$ Med Biol Res 41 (2008), 956-959.

[38] S.J. Padayatty, H. Sun, Y. Wang, H.D. Riordan, S.M. Hewitt, A. Katz, R.A. Wesley, and M. Levine, Vitamin C Pharmacokinetics: Implications for Oral and Intravenous Use, Ann Intern Med 140 (2004), 533-537.

[39] F.M. Palmer, D.C. Nieman, D.A. Henson, S.R. McAnulty, L. McAnulty, N.S. Swick, A.C. Utter, D.M. Vinci, and J.D. Morrow, Influence of vitamin $\mathrm{C}$ supplementation on oxidative and salivary IgA changes following an ultramarathon, Eur J Appl Physiol 89 (2003), 100-107.

[40] B. Palmieri and V. Sblendorio, Oxidative stress tests: overview on reliability and use. Part I, Eur Rev Med Pharmacol Sci 11 (2007), 309-342.

[41] R. Pazdro and J.R. Burgess, The role of vitamin E and oxidative stress in diabetes complications, Mech Ageing Dev 131 (2010), 276-286. 
[42] B. Rai, Salivary and Serum Vitamin C in Periodontal Disease, AMDS 2 (2008), 90-91.

[43] B. Rai, S. Kharb, R. Jain, and S.C. Anand, Salivary Vitamin E and $C$ in Lichen Planus, GJMS 6 (2008), 91-92.

[44] R.J. Reiter, D.-X. Tan, B. Poeggeler, A. Menendez-Pelaez, L.D. Chen, and S. Saarela, Melatonin As a Free Radical Scavenger: Implications for Aging and Age-Related Diseasesa, Ann N Y Acad Sci 719 (1994), 1-12.

[45] Y. Saral, B.K. Coskun, P. Ozturk, F. Karatas, and A. Ayar, Assessment of salivary and serum antioxidant vitamins and lipid peroxidation in patients with recurrent aphthous ulceration, Tohoku J Exp Med 206 (2005), 305-312.

[46] D.V. Sculley and S.C. Langley-Evans, Periodontal disease is associated with lower antioxidant capacity in whole saliva and evidence of increased protein oxidation, Clin Sci 105 (2003), 167-172.

[47] B. Sedighe, B. Maryam, A. Fahimeh, A. Somayyeh, and T.J. Bigom, Effect of vitamin $\mathrm{C}$ on salivary superoxide dismutase activity in smokers, $A J B \mathbf{1 0}$ (2011), 7267-7270.

[48] L. Selmeci, Advanced oxidation protein products (AOPP): Novel uremic toxins, or components of the non-enzymatic antioxidant system of the plasma proteome? Free Radic Res $\mathbf{4 5}$ (2011), 1115-1123

[49] R.S. Sohal, R.J. Mockett, and W.C. Orr, Mechanisms of aging:
An appraisal of the oxidative stress hypothesis, Free Radic Biol Med 33 (2002), 575-586.

[50] H. Su, M. Gornitsky, G. Geng, A.M. Velly, H. Chertkow, and H.M. Schipper, Diurnal variations in salivary protein carbonyl levels in normal and cognitively impaired human subjects, Age (Dordr) 30 (2008), 1-9.

[51] M. Takane, N. Sugano, H. Iwasaki, Y. Iwano, N. Shimizu, and K. Ito, New biomarker evidence of oxidative DNA damage in whole saliva from clinically healthy and periodontally diseased individuals, J Periodont 73 (2002), 551-554.

[52] T. Tomofuji, D. Ekuni, T. Sanbe, K. Irie, T. Azuma, T. Maruyama, N. Tamaki, J. Murakami, S. Kokeguchi, and T. Yamamoto, Effects of vitamin $\mathrm{C}$ intake on gingival oxidative stress in rat periodontitis, Free Radic Biol Med 46 (2009), 163-168.

[53] C.C. Tsai, H.S. Chen, S.L. Chen, Y.P. Ho, K.Y. Ho, Y.M. Wu, and C.C. Hung, Lipid peroxidation: A possible role in the induction and progression of chronic periodontitis, J Periodont Res 40 (2005), 378-384.

[54] D. Wei, X.L. Zhang, Y.Z. Wang, C.X. Yang, and G. Chen, Lipid peroxidation levels, total oxidant status and superoxide dismutase in serum, saliva and gingival crevicular fluid in chronic periodontitis patients before and after periodontal therapy, Aust Dent J 55 (2010), 70-78. 


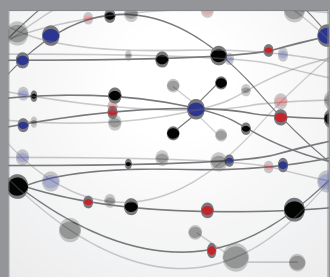

The Scientific World Journal
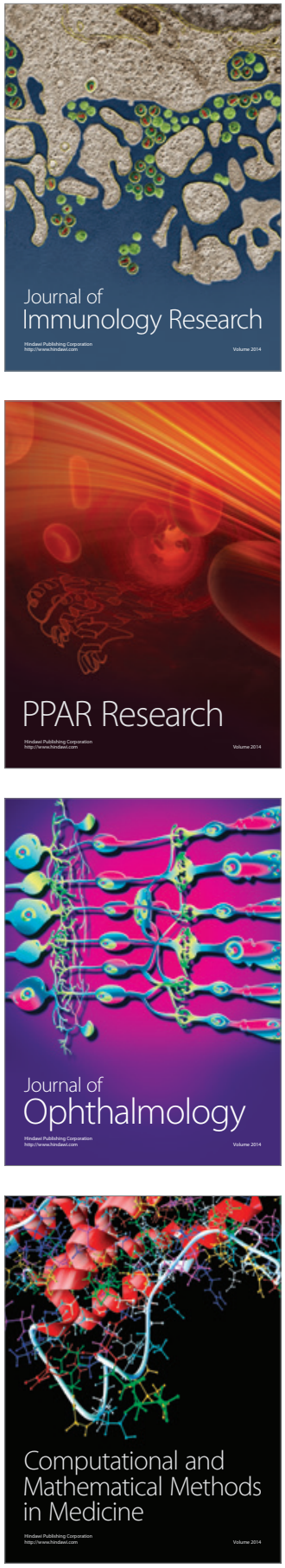

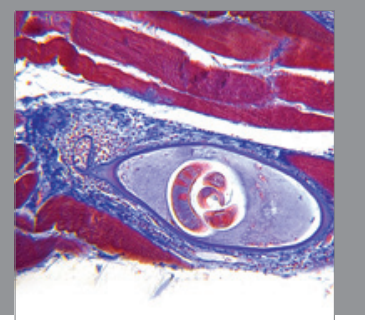

Gastroenterology

Research and Practice
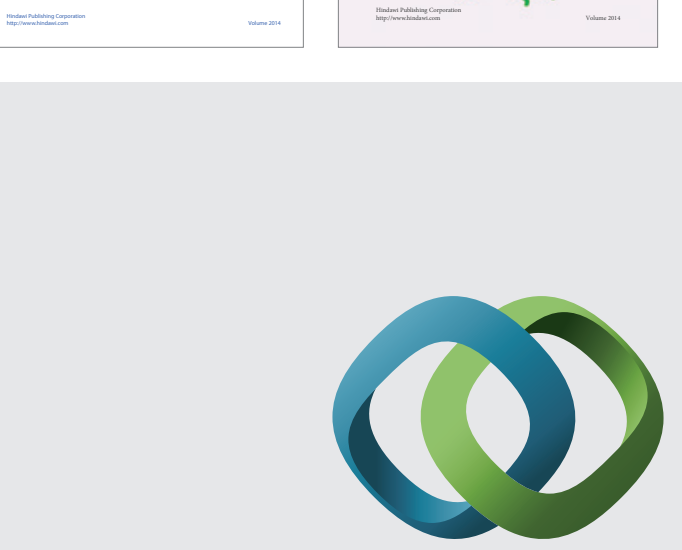

\section{Hindawi}

Submit your manuscripts at

http://www.hindawi.com
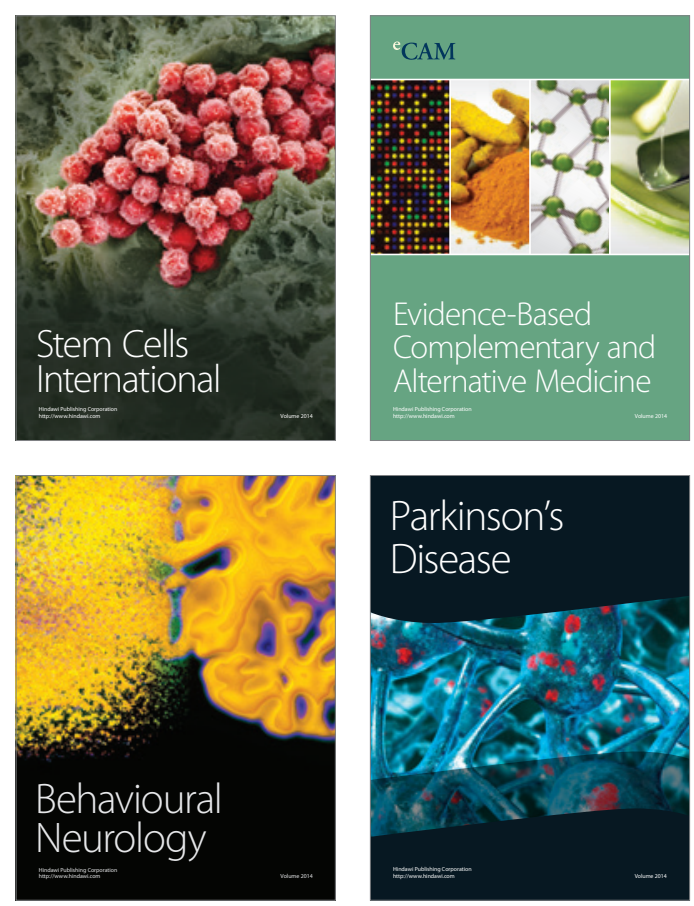

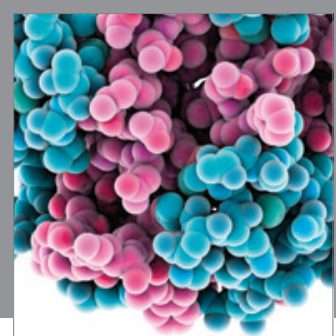

Journal of
Diabetes Research

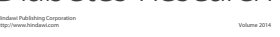

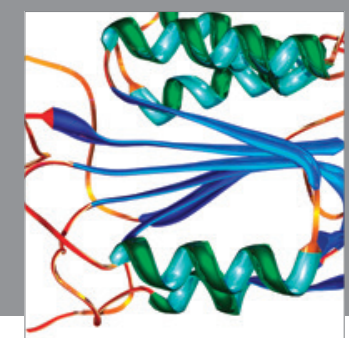

Disease Markers
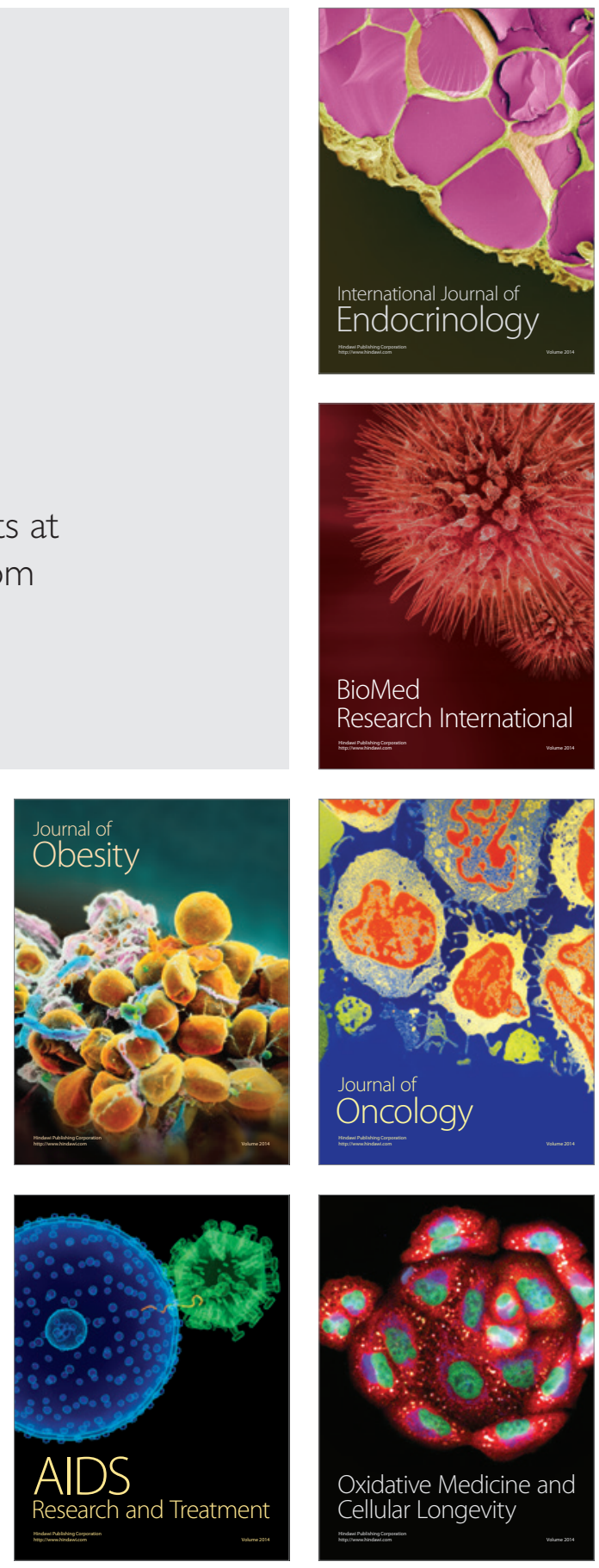